\title{
Textural approaches for vineyard detection and characterization using very high spatial resolution remote-sensing data
}

\author{
C. DELENNE* $\dagger$, S. DURRIEU $\dagger$, G. RABATEL $\dagger$, M. DESHAYES $\dagger$, J.S. BAILLY $\dagger$, C. LELONG $\dagger$, P. \\ COUTERON§ \\ $\dagger$ UMR TETIS, CEMAGREF - CIRAD - ENGREF, 34093 Montpellier, France \\ $\ddagger$ UMR ITAP CEMAGREF - ENSA - CIRAD, 34196 Montpellier, France \\ $\S$ French Institute of Pondicherry, PB 33, 605001 Pondicherry, India
}

(Received 00 Month 200x; In final form 00 Month 200x)

\begin{abstract}
Vine-plot mapping and monitoring are crucial issues in land management, particularly for areas where vineyards are dominant like in some French regions. In this context, the availability of an automatic tool for vineyard detection and characterization would be very useful. The objective of the study is to compare two different approaches to meet this need. The first one uses directional variations of the contrast feature computed from Haralick's cooccurrence matrices and the second one is based on a local Fourier Transform. For each pixel, a 'vine index' is computed on a sliding window. To foster large-scale applications, test and validation were carried out on standard very high spatial resolution remote-sensing data. $70.8 \%$ and $86 \%$ of the 271 plots of the study area were correctly classified using the cooccurrence and the frequency method respectively. Moreover, the latter enabled an accurate determination (less than $3 \%$ error) of interrow width and row orientation.
\end{abstract}

Keywords: Texture; Image analysis; Cooccurrence; Fourier Transform; Vineyard

\section{Introduction}

Thanks to the increased availability of remote sensing data and of more powerful computers, automatic analysis methods can be developed to build or update geographical databases for land management. Accurate digital mapping of vineyards for wine-growing regions such as Languedoc-Roussillon (France) could be extremely useful for many reasons. For example, these maps can be integrated within Geographical Information Systems (GIS) which can be used by winegrower cooperatives to improve the monitoring of quality compliance in areas registered in the list of Controlled Origin Denomination. The management of pollution, erosion and flood risks is another field that can take advantage of these maps. Indeed, these risks, depending on culture and soil surface condition, are worsened by mechanization and intensive cropping practices (Wassenaar et al., 2005; Vincini et al., 2004).

User demand usually concerns 1) locating vine plots and 2) identifying some characteristics that can be connected to cropping practices or crop quality (interrow width, orientation of rows, presence of grass between rows...).

Most vineyard related studies using remote sensing data meet the second requirement by detecting vine rows (Bobillet et al., 2003) for example, or by characterizing training mode (Wassenaar et al., 2002) or foliar density (Hall et al., 2003) for previously delimited plots. Those dealing with vineyard plots identification and delineation often use multi-spectral information on over-metric spatial resolution images, provided by satellites Landsat, Ikonos or airborne sensors (Rodriguez et al., 2006; Johnson et al., 2001; Gong et al., 2003). However, the increasing availability of Very High Spatial Resolution (VHSR) images offers a lot of new potential applications: the object shape and spatial structure are becoming more distinguishable, providing greater discrimination and characterization opportunities. Indeed, according to the ShannonNyquist theorem ${ }^{1}$, periodic patterns resulting from the spatial arrangement of vine plants (often in lines or grid), become perceptible with a spatial resolution that is at least twice as small as the pattern period.

\footnotetext{
${ }^{1}$ See any book on signal processing for more information about this theorem
} 
In the study area, like in many wine-growing regions, the minimum distance between two vine rows, is
about $1.5 \mathrm{~m}$; consequently, image spatial resolution should be lower than $0.75 \mathrm{~m}$. However, as they deal

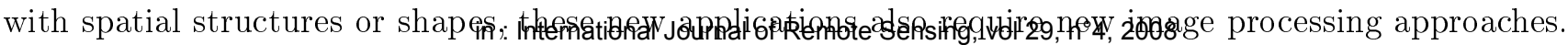

In a recent study (Warner et al., 2005), a classification algorithm based on an analysis of autocorrelograms was developed and tested using Ikonos panchromatic imagery of Granger (Washington). This method, although providing good results in the application presented, could hardly be generalized in older European wine-growing regions where the heterogeneity among researched patterns is high. Because of the periodic organization of vineyards, frequency analysis appears as a suitable approach for vine detection. Wavelet analysis presented in (Ranchin et al., 2001) is applied to $25 \mathrm{~cm}$ resolution images for vine/non-vine pixel classification. Using a plot basis validation, $78 \%$ of plots were accurately classified; but this approach is complex and needs significant user intervention. A Fourier Transform based analysis should be more straightforward and quite as effective since this tool is perfectly suited for oriented and periodic texture detection. Its efficiency has been demonstrated to characterize and monitor natural periodic vegetation (Couteron and Lejeune, 2001; Couteron, 2002). Wassenaar (Wassenaar et al., 2002) successfully used it for vine/non-vine classification and characterization of previously delimited plots on $25 \mathrm{~cm}$ resolution images. On a sample of 46 'extremely varied field patterns', vine/non vine classification was correct for all the plots and only five errors were encountered concerning training mode classification of the 41 vine plots. Moreover, this method gave a very precise (less than $1 \%$ error) estimation of interrow width and row orientation. Prat (Prat, 2002) employed a similar method to identify vine plots in an image. This one was first divided into small square windows (of $12.5 \mathrm{~m}$ side) on which five indices were deduced from Fourier spectrum and image radiometry. Then, a multidimensional supervised classification using maximum likelihood led to correct classification of $81 \%$ of vine pixels.

Other very popular approaches for textural analysis are based on Haralick's researches, according to whom 'the texture information in an image is contained in the overall or "average" spatial relationship which the gray tones in the image have to one another' (Haralick et al., 1973). He then introduced the 'gray-level spatial dependency' (cooccurrence) matrices, which had remained unused for many years as they were too time-consuming. With the amazing increase of computer power, cooccurrence became one of the most popular characterization tools because it is based on second order statistics, well suited for the description of textural properties, which the human eye is most sensitive to. A lot of studies have demonstrated its relevance for textural analysis (Chen et al., 1979) and its usefulness for many applications: urban planning (Morales et al., 2003), medicine (Smutek et al., 2003), scientific police (Verma et al., 2002), textile industry (Abdulhady et al., 2002)... and even remote-sensing for agro-forestry (Arvis et al., 2004).

The general objective of this work was to develop an automatic method for vineyard detection and characterization using very high spatial resolution remote-sensing data and without any a priori knowledge of the parcel plan. Indeed, this latter is not available in most European wine-growing regions and, when a georeferenced cadastre is available, it generally does not correspond to agricultural plots actually observable in the field. To foster large-scale applications, image used was a 'standard' orthophotography in natural colour, with a $50 \mathrm{~cm}$ spatial resolution, similar to data available on the whole French territory. In this paper, the relevance of cooccurrence based analysis is evaluated in comparison with a frequency approach to meet the need for vine plot detection. Moreover, characterizations of row orientation and/or interrow width, deduced from these approaches, are compared.

\section{Study area}

The study area is part of the La Peyne watershed $\left(110 \mathrm{~km}^{2}\right)$ and is located in the Languedoc-Roussillon region - France (Figure 1). This zone is representative of the French Mediterranean coastal plain with respect to geology, agricultural practices and vineyard management (Wassenaar et al., 2002). Two subsets, of $2 \mathrm{~km}^{2}$ and $1 \mathrm{~km}^{2}$, have been selected from this area near Roujan municipality $\left(43^{\circ} 30^{\prime} \mathrm{N}, 3^{\circ} 18^{\prime} \mathrm{E}\right)$. Despite a general decrease, vine cultivation is still predominant and covers about $70 \%$ of the 271 plots of the study area.

The diversity of agricultural practices in the study area leads to a great heterogeneity among vine plots (difference of vigour, grass between rows, missing vine trees...) which generally hampers the use of 


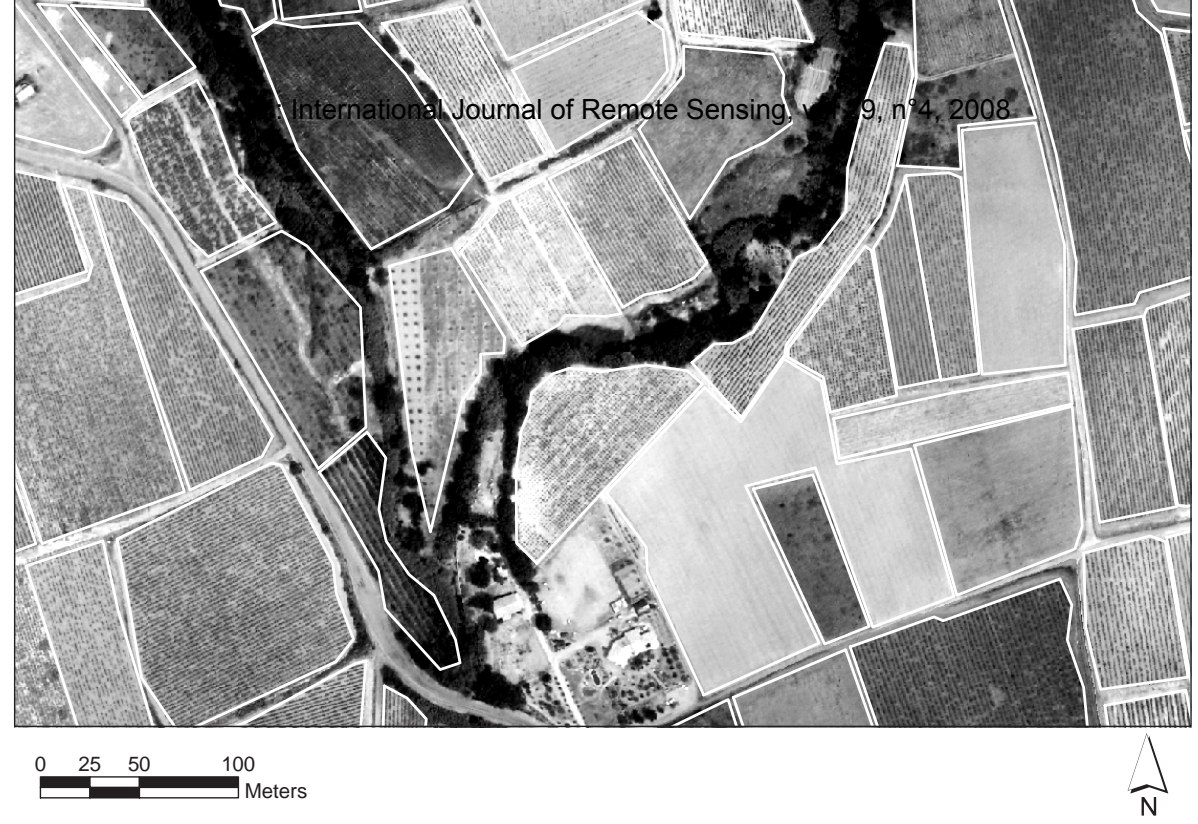

Figure 3. Zoom on the study area. All the plots of the study area have been surveyed and integrated with their characteristics in a geodatabase.

\section{Data acquisition}

Data acquisition was made during the first week of July 2005, when foliar development was such that both vine and soil were visible on aerial photographs. A digital camera was used aboard an Ultra Light Motorized (U.L.M.) to acquire photographs in natural colours (Red, Green and Blue). Images were geometrically corrected and georeferenced using $\operatorname{ArcGis}^{\circledR}$ (ESRI), mosaicked using ERDAS ${ }^{\circledR}$ Imagine (Leica Geosystem) and resampled to a $50 \mathrm{~cm}$ resolution. The resulting images have characteristics similar to those of the BDOrtho ${ }^{\circledR}$ coverage of the French geographic institute (IGN), which is widely used and covers almost all the French territory.

For result validation, ground-truth information was collected at the same time as image acquisition. Each of the 271 vine and non-vine plots of the site has been digitized in a GIS database (figure 3) which also contains information concerning land use and a series of characteristics for vine plots: training mode, interrow width, orientation, rough estimates of vine height and width, soil surface condition... Row orientation and interrow width were obtained by precise on-screen measurements: row orientation was measured with a $1^{\circ}$ precision and interrow width was calculated by dividing the width of the whole plot by the number of interrows.

\section{Textural analysis methods}

Both methods compared in this paper were implemented to calculate textural characteristics on the surrounding of each pixel using a sliding window.

\subsection{Cooccurrence analysis: use of Haralick's contrast feature}

The first method presented in this study has been developed from cooccurrence matrices defined in (Haralick et al., 1973). Element $p_{i, j}$ of each matrix $P_{d_{x}, d_{y}}$ contains the number of transitions from grey level $i$ to $j$ between two pixels of image $I$, distant from $d_{x}$ pixels in column and $d_{y}$ in line (equation 1 ): 
and 81 non vine plots using Red channel: a plot is well-classified if at least $75 \%$ of its pixels are well-classified, badly classified if less than $25 \%$ of its pixels are well-classified, not classified otherwise.

\begin{tabular}{|c|c|c|c|c|c|c|c|c|}
\hline & \multicolumn{7}{|c|}{$\begin{array}{l}\text { in: International Journal of Remote Sensing, vol } 29, n^{\circ} 4,2008 \text { FREQUENCY METHOD } \\
\text { CONTRAST METHOD }\end{array}$} & \multirow[b]{2}{*}{ Total } \\
\hline & vine & non vine & not classified & Total & vine & non vine & not classified & \\
\hline Vine & $72 \%$ & $7 \%$ & $21 \%$ & $100 \%$ & $89 \%$ & $5 \%$ & $6 \%$ & $100 \%$ \\
\hline Non vine & $1 \%$ & $68 \%$ & $31 \%$ & $100 \%$ & $2 \%$ & $79 \%$ & $19 \%$ & $100 \%$ \\
\hline
\end{tabular}

Because of the threshold chosen, both methods lead to a worse classification for non vine than for vine plots. The main cause of non detection is when the vine is too young i.e. less than three years old. Vegetation is thus not sufficiently developed for the rows to be visible on aerial photographs; consequently, these vine plots have good classification rates of only $26 \%$ and $47 \%$ for contrast and frequency methods respectively (see figure $9 \mathrm{~b}$ for an example).

Globally, results provided by contrast method are poorer than those provided by frequency method. For non vine plots, one explanation may be that contrast does not take into account the periodicity of patterns: a road, for example, can lead to a difference of contrast in two orthogonal directions that is as high as that of a vine but does not have a peak of frequency corresponding to vine interrow width. Figure 9a shows an example of non vine detected as vine by contrast method but not by frequency method. Likewise, confusion could theoretically appear between vine and orchards. However, since the sliding window size is adapted to vineyards interrow widths, vine index will be lower on orchards because their interrow widths are much larger.

For vine plots, results must be analyzed according to training mode. Indeed, goblet vines benefit from a good classification rate of only $49 \%$ using contrast method against $89.7 \%$ for 'adult' trellis vines (all goblet vines are adult). Likewise, frequency method leads to a good classification rate of $88.6 \%$ for goblet vines and $95.7 \%$ for 'adult' trellis vines.

For both methods, the poorer results obtained for goblet vines mainly have two origins which lead to a low visibility of soil between rows. Firstly, goblet vines are not stressed by wires and can grow freely in all directions; secondly, interrow widths of goblet vines are generally smaller than those of trellis vines (on the study area, $67 \%$ of the goblet vines have an interrow width lower than $160 \mathrm{~cm}$ against only $2 \%$ of the trellis vines).

Concerning goblet vine classification, the big difference $(39.6 \%)$ between methods is due to the fact that goblet vines are often planted on a square grid so that contrast is identical in both perpendicular directions, which hamper detection by the contrast method (see figure $9 \mathrm{c}$ for example). In fact, most goblet vines properly identified by the contrast method are pruned along one direction, which leads to a higher contrast in the perpendicular direction.

\subsection{Results of vine plot characterization}

Estimation of orientation and interrow width obtained by both methods are now examined.

With the contrast method, $78 \%$ of the plots correctly classified as vine have been allocated with the correct orientation class (among the four used). As evoked previously, defining more than four orientation classes cannot be considered with this resolution because it would imply, for contrast calculation, a distance too large in front of interrow width (figure 4). Indeed, if a $30^{\circ}$ class is sought, the distance in pixels needed to compute contrast feature will be at least $\left(d_{x}, d_{y}\right)=(5,3)$ i.e. an Euclidian distance of about $2.9 \mathrm{~m}$ (for $30.96^{\circ}$ and $10.98 \mathrm{~m}$ for $30.07^{\circ}$ ), larger than most of interrow width.

Figure 10a shows characterization results for well classified vine plots, using frequency method. Fourier Transform leads to more accurate results for vine row orientation. Indeed, between on-screen measurements and method estimation, an average absolute difference of $3.5^{\circ}$ was found, which is less than a $2 \%$ error. Moreover, error distribution is almost centered (figure 10b).

Interrow width calculation is also very precise with an average absolute difference of $6.2 \mathrm{~cm}$, i.e. about $3 \%$ error (see figure 10d for error distribution). The four outliers shown in figure 10c, concern two vine plots covered by grass only one interrow out of two, which leads to a pattern period twice as large as 


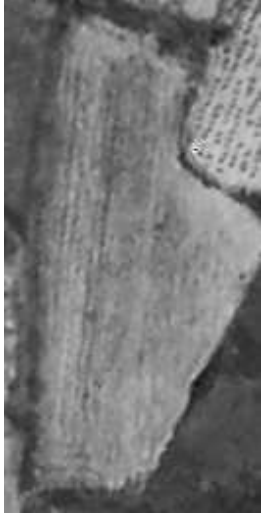

(a)

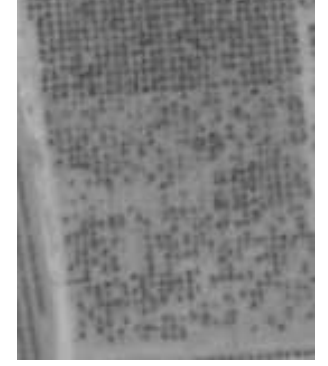

(c)

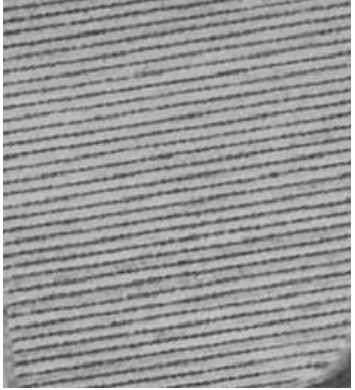

(d)

Figure 9. Examples of plots. a) Non cultivated plot recognized as vine by contrast method: an oriented pattern is visible but with no particular frequency. b) Very young trellis vine, badly classified by both methods: rows are hardly visible. c) Goblet vine classified as non vine by contrast method and well-classified (with $98 \%$ of pixels) by frequency method. d) Trellis vine, well classified by both methods.

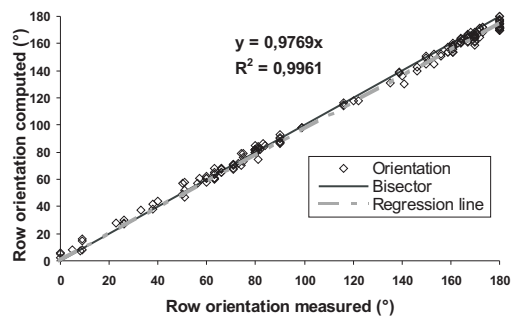

a)

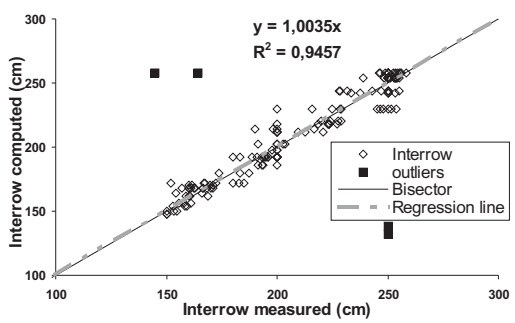

c)

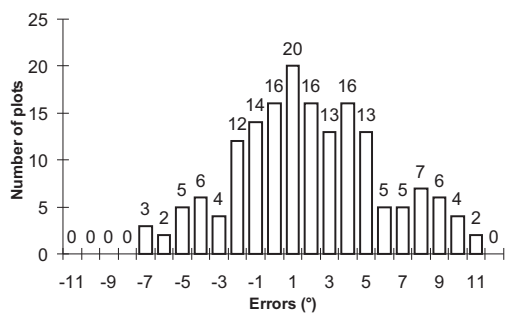

b)

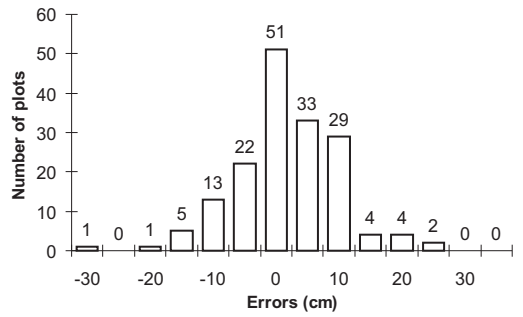

d)

Figure 10. Orientation and interrow characterization using frequency method. Right: comparison of retrieved characteristics and plot measurements; regression and bisector lines are almost confounded. Left: error distributions.

interrow width, and two vine plots ploughed between rows, for which interrow width determined is half on-screen measurement. Characterization results highly depend on the size of the calculation window (see below), which is why (Wassenaar et al., 2002), who applied the FFT algorithm on the entire plot, obtained $1 \%$ errors for both orientation and interrow width.

\section{Conclusion and discussion}

Two methods were compared for vineyard detection and characterization from aerial photograph presenting 'standard' characteristics. The first one was based on Haralick's cooccurrence analysis, which had been successfully tested on many applications but not yet for vineyard detection. The second one was based on Fourier analysis, a well-tried approach for periodic and oriented pattern recognition.

The originality of the proposed cooccurrence approach lies in the comparison of the contrast feature calculation in two orthogonal directions. However, this method leads to a poorer vine/non-vine classification 
- non-vine: contrast is sometimes higher in one direction than for its perpendicular, due to ploughing

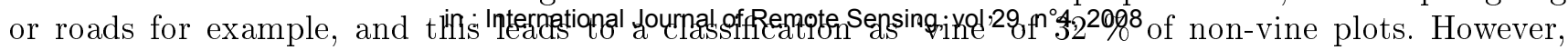
the resulting patterns have no particular frequency and are globally well-classified by frequency method (21\% of commission error).

- Goblet vines: with this training mode, when there is no privileged direction of pruning, contrast is the same in both perpendicular direction, which leads to a 'non-vine' classification of $51 \%$ of goblet vines. On the contrary, since their overall pattern is periodic, these plots are well-classified with the frequency method (only $21.4 \%$ of error).

The poorer results of goblet detection using both methods are strongly linked to the relation between the pattern period (interrow width) and the image resolution: the limit of the Shannon-Nyquist theorem is reached. However, this highlights the fact that a coarser resolution could be used in many other winegrowing regions, especially dry ones such as Castilla-la-Mancha in Spain, where interrow widths are up to three meters. These approaches could also be applied to orchards with other resolutions as long as the periodic pattern is visible.

In comparison with the contrast method, the frequency approach not only permits a better 'vine'/'nonvine' classification ( $86 \%$ against $70 \%$ ), but also a very precise estimation of row orientation and interrow width (2\% and $3 \%$ errors respectively) whereas only four classes of orientation could be defined and distinguished with an accuracy of $78 \%$ using the contrast feature.

In addition to the utility of characterization as such, orientation and interrow width estimation could be used to increase plot classification quality in prospect for a segmentation stage. On the one hand, they could be used to better separate detected plots; indeed, some plots, which are spatially very close, would be grouped within a same polygon unless they have different row orientation or interrow width. On the other hand, these characteristics could help the discrimination of badly classified non-vine plots; indeed, vine classified plots with no particular orientation or interrow width could be reclassified as non-vine.

In prospect, to meet the second user requirement, characteristics of row orientation and interrow width could also be used for an automatic detection of each vine row. This would enable the evaluation of more vineyard characteristics such as missing vine trees or soil surface condition (e.g. presence of grass between rows). Moreover, vine index could also be used on vine plots as an indicator of vine quality, since its intensity depends on the pattern contrast.

\section{Acknowledgements}

This work is part of two projects: BACCHUs European Project (http://www.bacchus-project.com) and MoBHYDIC project of the French national program of research in hydrology (PNRH). The authors thank C. Debain (CEMAGREF) for his contribution concerning the cooccurrence approach.

We wish to pay tribute to the late J.M. Robbez-Masson who died in a mountain accident. We are deeply grateful for his kindness, availability and for the very constructive discussions we shared with him concerning this work at meetings and on field trips.

\section{References}

Abdulhady, M., Abbas, H. Nassar, S., 2002, Fabric fault classification using neural trees. Proceedings of the IEEE International Conference on Systems, Man and Cybernetics, 6, 114-117.

Alonso, F., Algorri, M.E. Flores-Mangas, F., 2004, Composite index for the quantitative evaluation of image segmentation results. Proceedings of the 26th Annual International Conference of the IEE EMBS, San Francisco, USA.

Arvis, V., Debain, C., Berducat, M. Benassi, A., 2004, Generalization of the cooccurrence matrix for colour images: application to colour texture classification. Image Analysis and Stereology, 23, 63-72.

Bobillet, W., Da Costa, J.-P., Germain, C., Lavialle, O., Grenier, G., 2003, Row detection in high 
resolution remote sensing images of vine helds, European Conference on Precısıon Agrıculture, Berlın, 81-87.

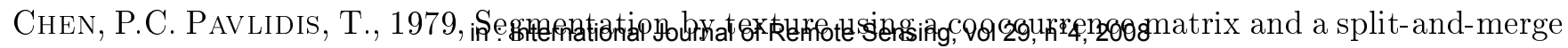
algorithm, Computer Graphes and Image Processing, 10, 172-182.

Couteron, P., 2002, Quantifying change in patterned semi-arid vegetation by Fourier analysis of digitised air photographs, International Journal of Remote Sensing, 23, 3407-3425.

Couteron, P. Lejeune, O., 2001, Periodic spotted patterns in semiarid vegetation explained by a propagation-inhibition model, Journal of Ecology, 89, 616-628.

Gong, P. Mahler, S.A., Biging, G.S Newburn, D.A., 2003, Vineyard identification in an oak woodland landscape with airborne digital camera imagery, International Journal of Remote Sensing, 24 (6), 13031315.

Hall, A., Louis, J., LAmB, D., 2003, Characterising and mapping vineyard canopy using high-spatialresolution aerial multispectral images, Computers and Geosciences, 29, 813-822.

Haralick, R.M., Shanmugam, K., Dinstein, I., 1973, Textural Features for Image Classification, IEEE Transaction on Systems, Man, and Cybernetics, 3 (6), 610-621.

Johnson, L.F., Roczen, D., Youkhana, S., 2001, Vineyard canopy density mapping with Ikonos satellite imagery, Presented at the Third International Conference on Geospatial Information in Agriculture and Forestry, Denver (Colorado), 5-7 November 2001.

Morales, D.I., Moctezuma, M., Parmiggiani, F., 2003, Urban and Non Urban Area Classification by Texture Characteristics and Data Fusion, International Geoscience and Remote Sensing Symposium (IGARSS), 6, 3504-3506.

PRAT, S., 2002, Caractérisation de végétations régulières par télédétection à très haute résolution spatiale: Application à l'analyse texturale de parcelles viticoles, DESS Statistique et Traitement du Signal, Université Blaise Pascal, Clermont-Ferrand.

Ranchin, T., Naert, B., Albuisson, M., Boyer, G., Astrand, P., 2001, An Automatic Method for Vine Detection in Airborne Imagery Using Wavelet Transform and Multiresolution Analysis, Photogrammetric Engineering and Remote Sensing, 67 (1), 91-98.

Rodriguez, J. R., Miranda, D., Alvarez, C. J., 2006, Application of Satillite Images to Locate and Inventory Vineyards in the Designation of Origin "Bierzo" in Spain, America Society of Agricultural and Biological Engineers, 49 (1), 277-290.

Smutek, D., Š́́ra, R., Sucharda, P., TJahjadi, T., Švec, M., 2003, Image texture analysis of sonograms in chronic inflammations of thyroid gland, Ultrasound in Medicine and Biology, 29 (11), 1531-1543.

Verma, M.S, Pratt, L., Ganesh, C., Medina, C., 2002, Hair-MAP: A prototype automated system for forensic hair comparison and analysis, Forensic Science International, 129 (3), 168-186.

Vincini, M., Frazzi, E., Assessment of erosion-related vineyards features in Tuscany by object-oriented classification of high resolution images Proceedings of Agro Environ 2004,20-24, October 2004, Udine (Italy).

Warner, T.A., Steinmaus, K., 2005, Spatial classification of orchards and vineyards with high spatial resolution panchromatic imagery, Photogrammetric Engineering and Remote Sensing, 71 (2), $179-187$.

Wassenafr, T., Robbez-Masson, J.-M., Andrieux, P., Baret, F., 2002, Vineyard identification and description of spatial crop structure by per-field frequency analysis, International Journal of Remote Sensing, 23 (17), 3311-3325.

Wassenafr, T., Andrieux, P., Baret, F., Robbez-Masson, J.-M., 2005, Soil surface infiltration capacity classification based on the bi-directional reflectance distribution function sampled by aerial photographs. The cases of vineyards in a Mediterranean area, Catena, 62, 94-110. 